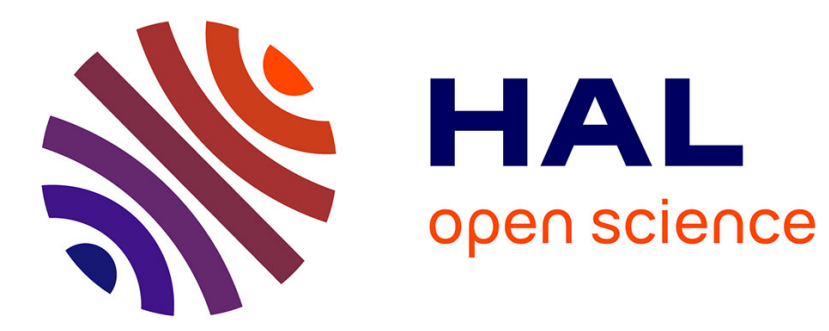

\title{
Pourquoi l'évaluation structurée du risque de récidive violente et sexuelle est une pratique marginale en France ? Revue systématique de la littérature
} Ingrid Bertsch, Thierry Pham, Christian Réveillère, Robert Courtois

\section{- To cite this version:}

Ingrid Bertsch, Thierry Pham, Christian Réveillère, Robert Courtois. Pourquoi l'évaluation structurée du risque de récidive violente et sexuelle est une pratique marginale en France ? Revue systématique de la littérature. Annales Médico-Psychologiques, Revue Psychiatrique, 2021, 10.1016/j.amp.2021.05.009 . hal-03265208

\section{HAL Id: hal-03265208 \\ https://hal.science/hal-03265208}

Submitted on 16 Sep 2021

HAL is a multi-disciplinary open access archive for the deposit and dissemination of scientific research documents, whether they are published or not. The documents may come from teaching and research institutions in France or abroad, or from public or private research centers.
L'archive ouverte pluridisciplinaire HAL, est destinée au dépôt et à la diffusion de documents scientifiques de niveau recherche, publiés ou non, émanant des établissements d'enseignement et de recherche français ou étrangers, des laboratoires publics ou privés. 


\section{Article original}

Pourquoi l'évaluation structurée du risque de récidive violente et sexuelle est une pratique marginale en France? Revue systématique de la littérature

Why is structured assessment of the risk of violent and sexual recidivism a marginal practice in France? A systematic review Ingrid Bertsch $^{124}$, Thierry Pham $^{3}{ }^{4}$, Christian Réveillère ${ }^{1}$ et Robert Courtois ${ }^{12}$

${ }^{1}$ Université de Tours, Département de psychologie, EE 1901 Qualipsy (Qualité de vie et santé psychologique), 37041 Tours cedex 1, France

${ }^{2}$ CHRU de Tours, CRIAVS Centre-Val de Loire, 37044 Tours cedex 9, France

${ }^{3}$ Centre de Recherche en Défense Sociale, 94 rue Despars 7500 Tournai, Belgique

${ }^{4}$ Université de Mons, UMONS, 20 Place du Parc, 7000 Mons, Belgique

Auteur correspondant : Ingrid Bertsch, Université de Tours, Département de psychologie, EE 1901 Qualipsy (Qualité de vie et santé psychologique), 37041 Tours cedex 1, France

Téléphone : 0218370598

Télécopie : 0218370590

Mail : i.bertsch@chu-tours.fr

Texte reçu le $1^{\text {er }}$ mars 2021 ; accepté le $1^{\text {er }}$ avril 2021

Résumé

En France, l'évaluation structurée du risque de récidive est peu développée face au jugement professionnel non structuré, pourtant moins valide.

Objectif. - Cet article propose une revue systématique de la littérature, répondant aux critères PRISMA, sur la perception et la pratique de l'évaluation structurée en France chez les professionnels concernés.

Méthode. - Les mots clés «Évaluation du risque » AND « récidive » AND « France » ont été recherchés dans Pubmed, PsycInfo, Cairn et ScienceDirect.

Résultats. - Les 23 articles obtenus dans les résultats montraient des craintes et peu d'avantages concernant l'évaluation structurée, la possibilité d'un système hybride et les quelques travaux français utilisant des échelles de risque. Ces réticences concernaient : $(i)$ le système judicaire français (notamment le tout actuariel) ; (ii) les professionnels évaluateurs (confusion des rôles 
entre professionnels); (iii) les personnes évaluées (risque de stigmatisation des infracteurs à haut risque) et (iv) les outils eux-mêmes (prédictivité modérée).

Discussion et conclusion. - La validité scientifique, clinique et les garanties éthiques des échelles structurées du risque sont pourtant suffisantes pour permettre leur développement en France. Quelques hypothèses sont avancées pour expliquer les réticences à leur généralisation en France.

Mots-clés : Auteur de violence sexuelle ; Échelle d'évaluation ; Évaluation du risque ; Récidive Revue de la littérature

\section{Abstract}

In France, the recidivism of offenders (both sexual and non-sexual violence) is a national concern as evidenced by the media and the succession of laws relating to the prevention of recidivism. Risk assessment can be performed using unstructured professional judgment which is unreliable or by structured risk assessment which are more reliable. Abroad, this structured assessment is considerably developed. In France, it gives rise to two opposing positions: (i) encouragement by public authorities and (ii) fear by a majority of professionals in the field.

Objective. - This article offers a systematic literature review, meeting PRISMA criteria, on the perception and practice of standardized evaluation in France by the professionals concerned.

Method. - The keywords "Risk assessment" AND "recurrence" AND "France" were searched in French and in English in the search engines Pubmed, PsycInfo, Cairn, and ScienceDirect.

Results. - The 23 articles obtained in the results highlighted (a) fears and few advantages regarding the use of structured evaluation, $(c)$ a possibility of a hybrid evaluation system in France and $(b)$ a report of some works already using scales of risk on French populations. This article focused mainly on the fears and perceived benefits of structured risk assessment. The reluctance has concerned both: $(i)$ the French judicial system (fear of a new penology based only on the actuarial approach and its consequences); (ii) the professional valuers (fear of a confusion of professional's roles in health and justice fields); (iii) the persons assessed (fear of a risk of stigmatization of high-risk patients concerning violence) and (iv) the tools themselves (fear of a poor reliability of the risk assessment). Very few articles highlighted their advantages: reliability, simplicity of use, good inter-judge fidelity, guideline for care, and specially an ability to overcom the insufficient basis for the evaluation resulting from unstructured clinical judgment. Results also suggested the possibility of a hybrid evaluation system in France, which could rely on risk scales, without neglecting the interview and the qualitative collected 
information that have to respect precise steps and allow the management of offenders. Finally, this paper presented the few French studies using risk scales mainly focused both in sexual offenders 'risks and associated factors, and showing a better productivity of these scales than an unstructured professional judgment.

Discussion and conclusion. - Fears related to the structured risk assessments are important. However, these scales may also supply a better vision of offenders. They require skills of professionals from various fields and allow both a fairer assessment of the offender and a penal system more adjusted. This perceived reluctance seems to be due to a poor knowledge of structured assessment the risk scales and a lack of research studies using them in France, where it is necessary to have a broad reflection on their development in the future.

Key words: Assessment tool; France; Literature paper; Recidivism; Perpetrator of sexual violence; Rating scale; Risk assessment

\section{Introduction}

La récidive des infracteurs est une préoccupation internationale. En France, sur la totalité des personnes condamnées en 2010, 40 \% avaient déjà un casier judiciaire au cours des huit années précédentes. Les auteurs d'infractions à caractère sexuel (AICS) ne sont pas, contrairement à la pensée commune, les infracteurs les plus récidivants : $12 \%$ (sur cinq ans) des personnes placées en bracelet électronique suite à une condamnation pour violence sexuelle ont récidivé en 2003 (vs $50 \%$ pour les faits de violence volontaire [30]). Ces taux de récidives vont dans le sens des données internationales, puisque la prévalence de la récidive sexuelle est de l'ordre de à $14 \%$ sur 5 ans et de $27 \%$ sur 20 ans [23].

Pour évaluer le risque de récidive, deux écoles existent : (a) le jugement professionnel non structuré (JPNS) (l'observation directe sans outil de mesure) très largement utilisé en France et $(b)$ les échelles de risque structurées très utilisées dans le monde. Aux États-Unis, l'évaluation du risque est devenue un élément clé de la réforme de la justice pénale et des peines fondées sur des preuves [8]. Plusieurs générations d'échelles ont vu le jour : (i) les échelles actuarielles (qui appliquent une méthode statistique permettant d'attribuer des points à des facteurs, corrélés à la récidive, afin de donner un score chiffré du risque de récidive de la personne évaluée ; [24], (ii) les échelles de jugement professionnel structuré (qui évaluent des facteurs de risque objectivés auxquels l'évaluateur accorde du poids selon ses observations ; 
[29]) et (iii) les échelles de jugement clinique intégré qui permettent d'évaluer le risque de récidive et qui aident à la mise en place d'un plan de gestion pour la réinsertion de l'infracteur [1]. Toutes s'appuient sur des facteurs dits «statiques » qui sont irréversibles, centrés sur le passé, évoluant peu dans le temps ou uniquement vers une majoration et/ou des facteurs dits «dynamiques» qui sont réversibles, évoluant dans le temps et permettant d'apprécier l'évolution de l'infracteur [38].

En France, l'évaluation structurée suscite globalement deux positionnements. D’un côté, les recommandations des instances publiques incitent à leur usage, soulignant leur caractère moins aléatoire et la nécessité de développer des travaux auprès de populations françaises [14,28]. Mais de l'autre, les professionnels de terrain se montrent globalement très réticents à leur utilisation, mettant en avant des critiques comme le manque de pertinence (pour $50 \%$ des experts interrogés), les contraintes techniques (30\%) et l'absence de validation des échelles de risque structurées sur des populations françaises $(10 \%)$ [10]. Cet article a pour objectif de mieux comprendre les enjeux liés aux perceptions négatives concernant la pratique de l'évaluation structurée du risque de récidive en France en se basant sur une revue systématique de la littérature et de proposer des pistes de réflexions sur cet état.

\section{Méthode}

Il s'agit d'une revue de littérature répondant aux critères PRISMA (Preferred Reporting Items for Systematic reviews and Meta-Analyses; [33]). La question de recherche est «Comment est perçue et utilisée l'évaluation structurée du risque de récidive par les professionnels en France. »

\subsection{Sources d'information et stratégie de recherche}

La recherche a été réalisée entre le 17 et le 19 février 2020. Les mots-clés « Évaluation du risque » AND « récidive » AND «France » ont été recherchés en français et en anglais sur des moteurs de recherche : «Pubmed», «PsycInfo » complétés par «ScienceDirect», ainsi que «Cairn » pour les revues de psychologie uniquement.

\subsection{Critères d'éligibilité}


Cette étude intègre tous les articles qui portaient sur le risque de récidive standardisé en général, sur l'évaluation structurée du risque, sur des populations d'infracteurs françaises, traitant d'une réflexion sur l'accueil ce type d'évaluation dans le système français et publiés entre 2005 et 2020.

\subsection{Sélection des articles et extraction des données}

Une des auteurs (IB) a procédé au premier tri des articles selon les critères d'éligibilité. Puis, un second tri a été réalisé par la lecture des résumés restants et enfin un dernier tri a été fait à la lecture des articles entiers restants (voir figure 1).

[Figure 1 à insérer environ ici]

\section{Résultats}

Les résultats traitent des craintes et des avantages perçus à l'évaluation structurée du risque, la possibilité d'un système d'évaluation hybride en France et les travaux français utilisant les échelles de risque de récidive. Le tableau 1 résume l'essentiel de chacune des 23 études retenues.

[Tableau 1 à insérer environ ici]

\subsection{Les craintes liées à l'évaluation structurée du risque de récidive}

Les craintes envers les échelles sont nombreuses et concernent à la fois la «philosophie » qui sous-tend l'évaluation structurée et les échelles elles-mêmes.

\subsubsection{Des craintes pour le système pénal français}

L'une des craintes fondamentales à l'arrivée des échelles de risque en France est l'intégration de la «philosophie actuarielle » qui amènerait à une «nouvelle pénologie » dédiée 
à la prise en charge (judiciaire et sanitaire) de la récidive et donc à risque d'être plus « sécuritaire» [25,26]. Pour certains auteurs comme Raoult [39], la récidive ne peut pas être une priorité pour la société car elle ne constitue pas une réalité des plus importantes. Cette philosophie actuarielle entrainerait des bouleversements trop inquiétants, notamment au niveau pénal, avec une pénologie plus sécuritaire qui se concentrerait sur la gestion du risque de comportements délictuels (le groupe) et non plus sur l'individu (la personne évaluée) [35]. Ceci réduirait le sujet à son appartenance à un groupe en fonction de son niveau de risque.

Sur le plan sanitaire, une philosophie actuarielle entraînerait des soins s'appuyant sur des facteurs objectifs, techniques, et attachés au risque. L'approche compréhensive et subjective du sujet (patient/ personne évaluée) serait alors perdue, avec deux conséquences irrémédiables [35,42] : (i) la perte d'une psychothérapie basée sur la rencontre intersubjective et (ii) le glissement du praticien vers un rôle d'expert uniquement. Dans cette perception, le praticien devrait se charger de mettre en place des soins uniquement centrés sur le risque de récidive et ses facteurs. Il ne pourrait s'intéresser à la totalité des difficultés que la personne rencontre et qu'il pourrait juger utile de changer ou d'améliorer. Finalement, cette philosophie actuarielle obligerait les thérapeutes à «soigner la souffrance de la société mise à mal par la violence du sujet délinquant $\gg[35$, p. 625].

\subsubsection{Des craintes pour le professionnel évaluateur}

L'histoire française est marquée par le rapprochement de la psychiatrie et de la justice (amenant au développement, toujours en cours, d'une psychiatrie légale). Ces liens étroits ont conduit à un rapprochement (voire une confusion) entre violence et troubles mentaux et l'investissement des experts psychiatres dans le prononcé du risque de dangerosité psychiatrique des infracteurs [35]. Ce lien s'est encore, récemment, renforcé puisqu'il est demandé aux experts psychiatres de se prononcer sur la dangerosité criminologique des infracteurs [35]. C'est sur ces évolutions que se cristallisent les craintes de l'évaluation structurée. On relève premièrement l'existence d'une crainte de confusion des domaines [35] et donc des rôles entre professionnels de la santé et de la justice. On relève aussi une crainte d'uniformisation rigide des pratiques des soins qui seraient plus centrées sur l'anticipation d'un comportement que sur le fonctionnement psychologique actuel du sujet. À ce titre, Voyer et Senon [42] rappellent que dans certains États américains, le score de l'évaluation du risque entraîne la détermination systématique de la peine. Partant de cette logique, l'évaluation structurée, réalisée par l'expert, sera uniquement centrée sur le risque de récidive. Si ce niveau de risque amène à un niveau de peine, alors l'expert peut se sentir à l'origine de la réponse 
pénale et penser qu'il se substitue au rôle de juge [17,35]. Enfin, une évaluation basée sur des facteurs précis entraîne la crainte d'une perte de «liberté de manœuvre » contraire au respect du Code médical qui préconise une indépendance professionnelle [42].

\subsubsection{Des craintes pour la personne évaluée}

Dans les modèles nord-américains, la philosophie actuarielle aboutit à une organisation de l'accompagnement (judiciaire et sanitaire) très différente. Le niveau de risque d'un infracteur va déterminer l'intensité de la prise en charge proposée. L'évaluation est donc, dans ce modèle, l'étape préalable à toute prise en charge [2]. Mais, le repérage des patients par leur niveau élevé de risque pourrait conduire à les isoler dans une étiquette de «patient dangereux », faisant craindre aux soignants une forme de régression des représentations longtemps combattues du « criminel fou » [35, p. 619]. De plus, cette stigmatisation pourrait ne jamais avoir de fin si les outils d'évaluation utilisés sont statiques et ne permettent pas d'apprécier suffisamment l'évolution des infracteurs $[19,42]$. Si l'évaluation a pour objectif d'aider à l'autonomisation du patient par la connaissance de ses propres facteurs de risque, cette même évaluation pourrait entraîner des temps de détention plus longs et donc un manque d'autonomie plus important [17]. Enfin, cette catégorisation des infracteurs pourrait conduire à « une exacerbation de la peur (des soignants et de la population) et l'acceptation tacite du sentiment diffus que chaque individu à risque potentiel de récidive est une bombe explosive en liberté » [17, p. 602].

\subsubsection{Craintes liées aux outils eux-mêmes}

Les échelles structurées de risque ont une image contrastée en France à la fois idéalisée (parce qu'elles rendent la situation d'évaluation plus simple à appréhender) et très critiquée [41]. Les échelles actuarielles sont les plus discutées parce qu'elles ont été les premières développées. Elles ne permettent pas de prédire le risque sur le court terme [35,42]; et les méthodes statistiques permettant de mettre en évidence leur efficacité ne seraient pas bonnes [6]. Par ailleurs, ces échelles qui sont composées de facteurs statiques ne seraient (i) pas conformes à la réalité clinique du sujet beaucoup plus dynamique ; (ii) elles ne permettraient pas d'apprécier l'évolution des infracteurs ( [17]et (iii) ne seraient pas une aide à leur prise en charge [20].

En matière de recherche, bien que certains soignants montrent une curiosité envers les échelles [16], d'autres mettent en avant l'impossibilité même de mener des recherches sur la récidive. Cette impossibilité serait due $(i)$ à la prépondérance des recherches sur la validation des échelles ne permettant pas de poser de questions empiriques essentielles à la recherche, (ii) 
au fait qu'aucune définition consensuelle de la récidive n'existe ou n'existerait et (iii) que cette recherche sur la récidive souffre de biais de confirmation des travaux successifs n'apportant aucun débat et surestimant l'efficacité des échelles [39].

Sur le plan pratique, l'intégration de ces échelles dans la culture française semble compliquée selon certains auteurs [35]. Le manque de données sur des populations françaises fait craindre une impossibilité d'utilisation de ces échelles en France [35]. De surcroît, une formation spécifique pour chaque échelle est nécessaire, voire obligatoire rendant leur utilisation plus « coûteuse » pour les professionnels [21].

\subsection{Les avantages offerts par l'utilisation de l'évaluation structurée du risque de récidive}

\subsubsection{L'inefficacité du jugement clinique non structuré dans l'évaluation du risque}

Si l'évaluation du risque existe déjà en France dans de nombreuses expertises, elle fait le plus souvent appel au JPNS, pourtant peu efficace car s'appuyant sur des heuristiques [18,21,31]. Celles-ci conduisent à des biais qui expliquent le caractère erroné du JPNS. Ces biais peuvent être émotionnels et/ou cognitifs. Ils amènent les évaluateurs à ( $i$ ) estimer le poids des facteurs de risque en fonction de leur vécu personnel, (ii) à confondre saillance et fréquence des événements, (iii) à avoir une vision stéréotypée du monde et (iv) à faire correspondre les événements nouveaux avec la représentation qu'ils se font déjà de la situation [21]. Ces biais augmentent le plus souvent la perception d'un risque qui impacte la situation pénale/sociale et psychologique de l'infracteur et exacerbent une crainte chez les professionnels [17].

\subsubsection{Les avantages liés aux échelles elles-mêmes}

Les échelles de risque, qu'elles soient actuarielles ou dynamiques, présentent divers avantages. Premièrement, elles permettent une évaluation du risque de meilleure qualité. Elles offrent la possibilité à l'évaluateur de préciser son avis en se basant sur l'ensemble des facteurs positivement corrélés au risque de récidive [35]. Elles amènent une évaluation stable, même pour des évaluateurs différents (bonne fidélité inter-juge) [42]. Elles ont une simplicité et une rapidité d'exécution qui rendent l'évaluation simple d'utilisation [42]. Deuxièmement, elles sont sources de partage pour les professionnels évaluateurs. Par leur aspect objectif, elles permettent une discussion entre des membres d'une équipe, avec des équipes extérieures sanitaires et/ou judiciaires $[11,34,42]$. Troisièmement, elles sont une aide à la prise en charge des infracteurs. Les échelles plus récentes, par leur introduction de facteurs dynamiques, vont 
présenter un intérêt clinique permettant aux professionnels d'affiner l'évaluation, d'apprécier l'évolution de l'infracteur et de proposer une prise en charge adaptée [21, 42].

\subsubsection{Quelles conséquences à une évaluation erronée du risque de récidive?}

En matière d'évaluation prédictive, la non-utilisation d'outils structurés amène à des prédictions mauvaises, qui surévaluent le risque d'un infracteur (faux positifs). Dans une volonté éthique, il semble normal que chaque infracteur ait le droit à une évaluation objective et rigoureuse puisque celle-ci impactera sa vie future [11]. En matière de soins, dans les pays nord-américains, la prévention de la récidive est souvent basée sur le modèle du RisqueBesoins-Réceptivité [21]. Ce modèle est le plus efficace pour diminuer le risque de récidive d'un infracteur [2]. Le «Risque » représente la priorité qui doit être donnée aux interventions. Les patients présentant les risques le plus élevés doivent bénéficier d'une prise en charge plus intensive. Le «Besoin » représente la nécessité de centrer la prise en charge sur des facteurs criminogènes spécifiques de l'infracteur évalué. La «Réceptivité » représente l'utilisation d'une intervention thérapeutique structurée et opérationnelle, plutôt d'orientation cognitive et comportementale. Le modèle RBR montre que la prévention de la récidive doit répondre à des principes globaux et que son efficacité dans la diminution du risque est bonne lorsque les trois principes sont respectés [2]. Il peut exister un danger à ne pas suivre ces principes en termes d'inefficacité de la prise en charge sur la récidive si la réponse n'est pas adaptée au niveau de risque (interventions trop peu intenses auprès d'infracteurs à haut risque ou au contraire, réponse trop intense auprès d'infracteurs à faible niveau de risque). Dans ce dernier cas, le risque de récidive peut même augmenter à moyen ou long terme.

\subsubsection{Le développement de nouvelles échelles}

Les échelles actuarielles de première génération ont fait l'objet de nombreuses critiques. Mais l'évolution des échelles s'est souvent faite en réponse aux critiques qu'elles ont reçues par la communauté et qui ont permis de passer d'un intérêt pour la prédiction pure à des préoccupations cliniques. Ceci est illustré par l'évolution des échelles actuarielles vers le jugement clinique intégré et le développement du jugement clinique structuré [21]. L'Inventaire de niveau de service et de gestion des cas [LS/CMI ; 1] est un exemple d'outil de jugement clinique intégré permettant à la fois une évaluation du risque et une aide à la création d'un plan de gestion pour l'évaluer. À l'aide de onze sections, complétées sur la base de l'évaluation du dossier correctionnel et d'entretiens semi-structurés, il aide à l'évaluation du risque général. Le Protocole de risque de violence sexuel (Risk for Sexual Violence Protocol : RSVP ; [27]) est 
un outil de jugement clinique structuré qui permet la prédiction du risque en utilisant des facteurs identifiés, mais pour lesquels l'évaluateur va pouvoir donner du poids en fonction de ses observations. À l'aide de cinq sections, complétées sur la base de l'évaluation du dossier correctionnel et d'entretiens semi-structurés, il aide à la prédiction du risque sexuel.

\subsection{Vers un système d'évaluation hybride?}

L'implantation d'un modèle étranger en France doit répondre à des conditions : $(i)$ être un besoin pour les utilisateurs, (ii) être implanté par les professionnels de terrain, (iii) naître dans des conditions matérielles adéquates et (iv) s'adapter à la culture d'accueil [21]. L'évaluation devra respecter des étapes précises. Il est indispensable qu'elle soit composée d'un pan quantitatif (avec des échelles structurées de risque) et d'un pan qualitatif (avec entretien) $[9,42]$. Elle devra avoir comme fonction d'adapter la prise en charge des infracteurs à leurs sorties de détention : (i) un risque faible entraînera un «suivi médico-psychologique souple », (ii) un risque modéré entraînera une injonction de soins dans le cadre d'un suivi socio-judiciaire (avec possibilité d'un traitement médicamenteux), et (iii) un risque fort entraînera un suivi médico-psychologique associé à des mesures de contrainte (comme des visites à domicile du conseiller pénitentiaire de probation) [9]. Enfin, la création et le développement de ce système d'évaluation devront être encadrés (par exemple par un comité d'éthique et de réflexion) [17]. Sa pratique devra exercée par des professionnels ayant les compétences requises et être discutée en équip [21,36, 42].

\subsection{Des travaux en population française}

Les quelques travaux s'intéressant au risque sur des populations françaises sont principalement centrés sur le risque de récidive sexuelle $[15,40]$ et violent $[5,37,40]$ des Auteurs d'Infraction à Caractère Sexuels (AICS) et beaucoup moins au risque général [4].

Ils montrent que dans une population d'AICS, le risque de récidive sexuelle (évalué avec la Static-99) est plus présent chez (i) les AICS d'adultes et les AICS « mixtes » (ayant des victimes adultes et enfants) que ceux n'ayant que des victimes mineures, et (ii) chez les AICS d'enfants extrafamiliaux face aux intrafamiliales [15]. Le risque de récidive violente est plus important chez les AICS que les homicidaires (évalué par l'échelle Violence Risk Assessment Guide - VRAG) [40]. Des facteurs sont positivement corrélés avec les risques de récidive sexuelle et violente : $(i)$ l'âge précoce d'entrée dans délinquance (VRAG, SORAG (Offender 
Risk Assessment Guide), Static-99, PCL-R (Psychopathy Checklist-Revised)), (ii) les antécédents judiciaires (SORAG, Static-99, PCL-R ;[40]), (iii) l'attribution externe des faits [7,19] et (iv) certains troubles psychiatriques comme des addictions (HCR-20, Historical, Clinical, Risk-20), des troubles de la personnalité narcissique, antisociale (HCR-20, PCL-R) et borderline (HCR-20) [37]. Certains facteurs sont associés négativement avec les risques : l'empathie (Static-99), l'estime de soi (Static-99), l'anxiété généralisée et le risque suicidaire (HCR-20, PCL-R). La validité convergente a été prouvée entre deux échelles de risque de récidive violente (la HCR-20 et la PCL-R) [37]. Enfin, une étude montre que l'avis des experts sans échelle est globalement concordant aux échelles pour diagnostiquer la psychopathie, mais pas pour évaluer la dangerosité souvent surestimée [40].

\section{Discussion}

Cette revue de la littérature s'est intéressée à la perception et l'utilisation de l'évaluation structurée du risque de récidive par les professionnels en France. Elle a mis en évidence de nombreuses craintes et relativement peu d'avantages liés à l'arrivée de ces échelles. Les craintes exprimées concernent à la fois le système pénal, les évaluateurs, les personnes évaluées et les échelles de risque elles-mêmes. Les avantages retrouvés sont une évaluation plus juste, plus stable pour les personnes évaluées et les professionnels évaluateurs, la possibilité de partager ces évaluations en équipe ou entre professionnels, une gestion de la récidive plus efficace et le fait que les nouvelles échelles intègrent l'avis de l'évaluateur et guident la prise en charge.

Cette étude montre que les craintes sont bien plus présentes que les avantages. Pourtant d'autres bénéfices sont perceptibles pour l'utilisation de l'évaluation structurée du risque. Concernant la perte de subjectivité dans l'évaluation, Benbouriche et al. [3] rappellent que la prévention de la récidive n'est pas stigmatisante, car l'évaluation du risque est basée sur les vulnérabilités des infracteurs, mais aussi leurs ressources. Les échelles, plus récentes permettent une évaluation plus positive des infracteurs en mesurant la présence de facteurs de protection. C'est le cas de la SAPROF (Structured Assessment of Protective Factors ; [12]) qui met en avant les ressources personnelles, sociales et institutionnelles des infracteurs [13]. D'autre part, il existe une confusion importante entre «mesure » et «évaluation » : l'évaluation n'est pas l'association entre des chiffres et des individus gérés par des règles précises, mais «un processus dans lequel plusieurs informations, de nature qualitative et quantitative, sont prises en compte afin de bien comprendre le fonctionnement d'une personne au travers de la considération de dimensions individuelles et situationnelles » [3, p.314]. Concernant la crainte 
de confusion des rôles, il semble important de rappeler que la prédiction du risque est une tâche complexe qui fait intervenir de nombreux domaines (judiciaire, social, psychologique et médical). L'évaluation d'une même personne s'intéressera à des facteurs judiciaires (comme les antécédents de risque), mais aussi sociaux (comme les finances), psychologiques (comme la personnalité) et psychiatriques (comme la présence d'une maladie psychiatrique). Elle nécessite donc l'implication de chacun des professionnels avec ses compétences spécifiques [41]. Concernant la stigmatisation des personnes évaluées qui serait liée à une forme d'étiquetage des infracteurs en fonction de leur niveau de risque, on peut déjà rappeler que les échelles de risque utilisent des critères objectifs amenant à une diminution de faux-positifs (très présents chez les évaluateurs utilisant le JPNS) et qui abaissent la proportion d'infracteurs jugés à haut risque de récidive, mais qui pourtant ne récidiveront pas [11,19]. Ceci minore la pression sécuritaire. D'autre part, les nouvelles échelles de risque amènent à une évaluation plus dynamique permettant de percevoir l'évolution de l'infracteur. Enfin concernant la fiabilité des échelles, il faut rappeler que celles-ci n'ont jamais été garantes d'un risque zéro et qu'elles doivent être considérées pour ce qu'elles sont, c'est-à-dire une aide à l'évaluation. Par exemple, dire qu'elles proposent une mauvaise prédiction à court terme n'a pas de sens, puisqu'elles évaluent un risque sur le long terme. On retiendra surtout que ces échelles structurées restent de meilleurs prédicteurs que le JPNS [32].

Finalement, les critiques semblent surtout refléter $(i)$ la méconnaissance des échelles (leur construction, leurs qualités psychométriques, leur utilisation et leur évolution) et (ii) le manque de recherches françaises sur la question. La formation aux échelles et leur accès par les professionnels doivent se développer. Bien que la formation représente un investissement en termes de coûts et du temps passé, elle permet une meilleure connaissance et surtout une utilisation plus rigoureuse des échelles [22]. L’Université (notamment les filières de psychiatrie et de psychologie légales) pourrait poursuivre son travail d'intégration des connaissances actualisées sur les échelles de risque. Le développement de la recherche en France sur les échelles de risque est aussi nécessaire et permettra de contribuer à cet axe.

Il serait intéressant qu'une réflexion nationale soit menée sur l'évaluation du risque impliquant tous les domaines des professionnels de terrain concernés et qu'elle permette d'apprécier les besoins des professionnels (dans le respect de leur rôle respectif) et les aménagements nécessaires pour l'adaptation des modèles étrangers à la culture et au système français.

Conflit d'intérêts : Les auteurs déclarent ne pas avoir de liens d'intérêts. 


\section{Références}

[1] Andrews DA, Bonta JL, Wormith JS. The Level of Service/Case Management Inventory (LS/CMI). Crime \& Delinquency 2004;7-27.

[2] Benbouriche M, Vanderstukken O. Guay JP. (). Les principes d'une prévention de la récidive efficace: Le modèle Risque-Besoins-Réceptivité. Pratiques Psychologiques 2015;21:219-34. https://doi.org/10.1016/j.prps.2015.05.002

[3] Benbouriche M, Ventejoux A, Lebougault M, Hirschelmann A. L'évaluation du risque de récidive en France: Expérience et Attitudes des Conseillers Pénitentiaires d'Insertion et de Probation. Revue Internationale de Criminologie et de Police Technique et Scientifique 2012;305-18.

[4] Bertsch I, Marcel D, Larroque I, Chouli B, Prat S, Courtois R. Prévenir la récidive de violence sexuelle en s'intéressant aux conditions de sortie de détention des auteurs d'infractions à caractère sexuel en France? [Preventing recidivism in sexual offenders by focusing on the conditions of release from custody in France?]. La Presse Médicale 2017;46. https://doi.org/10.1016/j.lpm.2017.03.016

[5] Bertsch I, Pham TH, Reveillère C, Courtois R. Évaluation du risque de récidive des auteurs d'infraction à caractère sexuel. Ann Méd Psychol 2017;175:294-6. https://doi.org/10.1016/j.amp.2017.01.015

[6] Bessoles P. Criminalité sexuelle et récidive. In Victimologie et criminologie. Approches cliniques 2004:75-88). Champ social. https://www.cairn.info/victimologie-etcriminologie-approches-cliniques--2913376436-page-75.htm?contenu=resume

[7] Blatier C, Sellon C, Gimenez C, Paulicand M. The assessment of incarcerated sexual delinquents' risk of recidivism. European Review of Applied Psychology 2016;66:18998. https://doi.org/10.1016/j.erap.2012.03.001

[8] Casey PM, Warren RK, Elek JK. Using offender risk and needs assessment information at sentencing: Guidance for courts from a national working group. National Center for State Courts; 2011.

[9] Coutanceau R. Dangerosité criminologique et prévention de la récidive: Évaluer la dangerosité sans stigmatiser l'homme. L'information Psychiatrique 2012;8:641-6. https://doi.org/10.3917/inpsy.8808.0641

[10] Crampagne S. L'évaluation de la dangerosité dans le cadre de l'expertise psychiatrique pénale [Thèse de doctorat en médecine]. Faculté de médecine de Grenoble; 2013.

[11] Dailliet A. Approche expertale évolutive et comparative: Un point de vue de défense 
sociale ? L'information psychiatrique 2008;84:35-9.

[12] de Vogel V, de Ruiter C, Bouman Y, de Vries Robbe M.SAPROF. Guidelines for the assessment of protective factors for violence risk (Forum Educatief) 2009.

[13] Deković M. Risk and Protective Factors in the Development of Problem Behavior During Adolescence. Journal of Youth and Adolescence 1999;28:667-85. https://doi.org/10.1023/A:1021635516758

[14] Delarue JM, et al. Auteurs de violences sexuelles: Prévention, évaluation, prise en charge. Rapport de la Commission d'audition du 17 juin 2018. Paris : Audition Publique, 14-15 juin 2018, Auteurs de Violences Sexuelles : Prévention, évaluation, prise en charge.

[15] Ducro C, Pham TH, Saloppé X, Chudzick L, Réveillère C. Évaluation du risque de récidive d'agresseurs sexuels français: Statique-99 et Sorag. Ann Méd Psychol 2012;170:106-8. https://doi.org/10.1016/j.amp.2012.01.001

[16] Goumilloux R. Prédicteurs de risque de récidive dans le cadre de suivis sociojudiciaires avec injonction de soins. L'information psychiatrique 2016;92:641-8.

[17] Gravier B, Moulin V, Senon JL. L'évaluation actuarielle de la dangerosité: Impasses éthiques et dérives sociétales. L’information Psychiatrique 2012;8:599-604. https://doi.org/10.3917/inpsy.8808.0599

[18] Grove WM, Zald DH, Lebow BS, Snitz BE, Nelson C. Clinical versus mechanical prediction: A meta-analysis. Psychological Assessment 2000;12:19-30.

[19] Guay JP. Évaluer le risque de récidive: Un état de la recherche et des principaux enjeux pratiques. Conférence de consensus; 2012.

[20] Guay JP, Benbouriche M, Parent G. L'évaluation structurée du risque de récidive des personnes placées sous-main de justice : Méthodes et enjeux. Pratiques Psychologiques 2015;21:235-57. https://doi.org/10.1016/j.prps.2015.05.005

[21] Guay JP, Lafortune D. L'évaluation du risque de récidive et l'intervention basée sur les données probantes: Les conditions nécessaires à l'implantation de méthodes structurées d'évaluation et d'intervention efficaces. Pratiques Psychologiques 2015;21:293-303. https://doi.org/10.1016/j.prps.2015.05.004

[22] Guay JP, Parent G. L'expertise pénale psychologique et psychiatrique. In Les échelles actuarielles. Paris: Dunod; 2014. P 109-25. Dunod. https://www.cairn.info/l-expertisepenale-psychologique-et-psychiatrique--9782100709489-page-109.htm

[23 Hanson RK, Morton KE, Harris AJR. Sexual Offender Recidivism Risk. Annals of the New York Academy of Sciences 2003;989:54-166. https://doi.org/10.1111/j.1749- 
6632.2003.tb07303.x

[24] Hanson RK, Morton-Bourgon KE. The accuracy of recidivism risk assessments for sexual offenders: A meta-analysis of 118 prediction studies. Psychological Assessment 2009;21:1-21. https://doi.org/10.1037/a0014421

[25] Harcourt BE. Surveiller et punir à 1'age actuariel 2011;35:5-33.

[26] Harcourt BE. Surveiller et punir à l'âge actuariel. Généalogie et critiques (Partie II). 2011;2:63-1954.

[27] Hart SD, Boer DP. Structured professional judgment guidelines for sexual violence risk assessment: The Sexual Violence Risk-20 (SVR-20) and Risk for Sexual Violence Protocol (RSVP). In R. K. Otto \& K. S. Douglas, Handbook of violence risk assessment 2010;269-94). Routledge, Taylor \& Francis Group.

[28] Haute Autorité de Santé. De la prévention de la récidive [Conférence de consensus] 2013.

[29] Hogan NR, Olver ME. Assessing risk for aggression in forensic psychiatric inpatients: An examination of five measures. Law and Human Behavior 2016;40:233-43. https://doi.org/10.1037/lhb0000179

[30] Josnin R. Une approche statistique de la récidive des personnes condamnées. Infostats Justice 2014; 127.

[31] Loza W, Dhaliwal GK. Predicting violence among forensic-correctional populations: The past 2 decades of advancements and future endeavors. Journal of Interpersonal Violence 2005;20:188-94. https://doi.org/10.1177/0886260504268767

[32] Meehl PE. Clinical versus statistical prediction: A theoretical analysis and a review of the evidence (Minnesota university) 1954.

[33] Moher D, Liberati A, Tetzlaff J, Altman DG, PRISMA Group. Preferred reporting items for systematic reviews and meta-analyses: The PRISMA statement. PLoS Medicine 2009;6(7). https://doi.org/10.1371/journal.pmed.1000097

[34] Mouchet-Mages S, Vittoz A, Braq-Leca H, Abderrahmane N, Pariat B, Debeaulieu F, et al. Apport des outils de jugement clinique structuré pour la mise en place d'un programme de soins individualisé des auteurs de violences sexuelles. Ann Méd Psychol 2018;176:100-2. https://doi.org/10.1016/j.amp.2017.11.005

[35] Moulin M, Palaric R, Gravier B. Quelle position professionnelle adopter face à la diversité des problèmes posés par l'évaluation des dangerosités? L’information Psychiatrique 2012;8:617-29. https://doi.org/10.3917/inpsy.8808.0617

[36] Niveau G. Évaluation de la dangerosité et du risque de récidive. Paris: L’Harmattan; 
2011.

[37] Pham TH, Chevrier I, Nioche A, Ducro C, Réveillère C. Psychopathie, évaluation du risque, prise en charge. Ann Méd Psychol 2005;163:878-81.

[38] Quinsey VL, Rice ME, Harris GT. Actuarial Prediction of Sexual Recidivism. Journal of Interpersonal Violence 1995;10:85-105.

[39] Raoult S. L'évaluation du risque de récidive: L'expert, le politique et la production du « chiffre ». Revue de science criminelle et de droit penal compare 2014;3:655-67.

[40] Rio M, Letto N, Butucea C. Usage exploratoire d'outils d'évaluation structurée du risque de récidive en France. Ann Méd Psychol 2017;175:287-9. https://doi.org/10.1016/j.amp.2017.01.014

[41] Vanderstukken O, Lacambre M. Dangerosité, prédictivité et échelles actuarielles: Confusion ou détournement? L'information psychiatrique 2011;87:549-50.

[42] Voyer M, Senon JL. Présentation comparative des outils d'évaluation du risque de violence. L'information psychiatrique 2012;88:445-53. 
Figure 1. Diagramme de flux
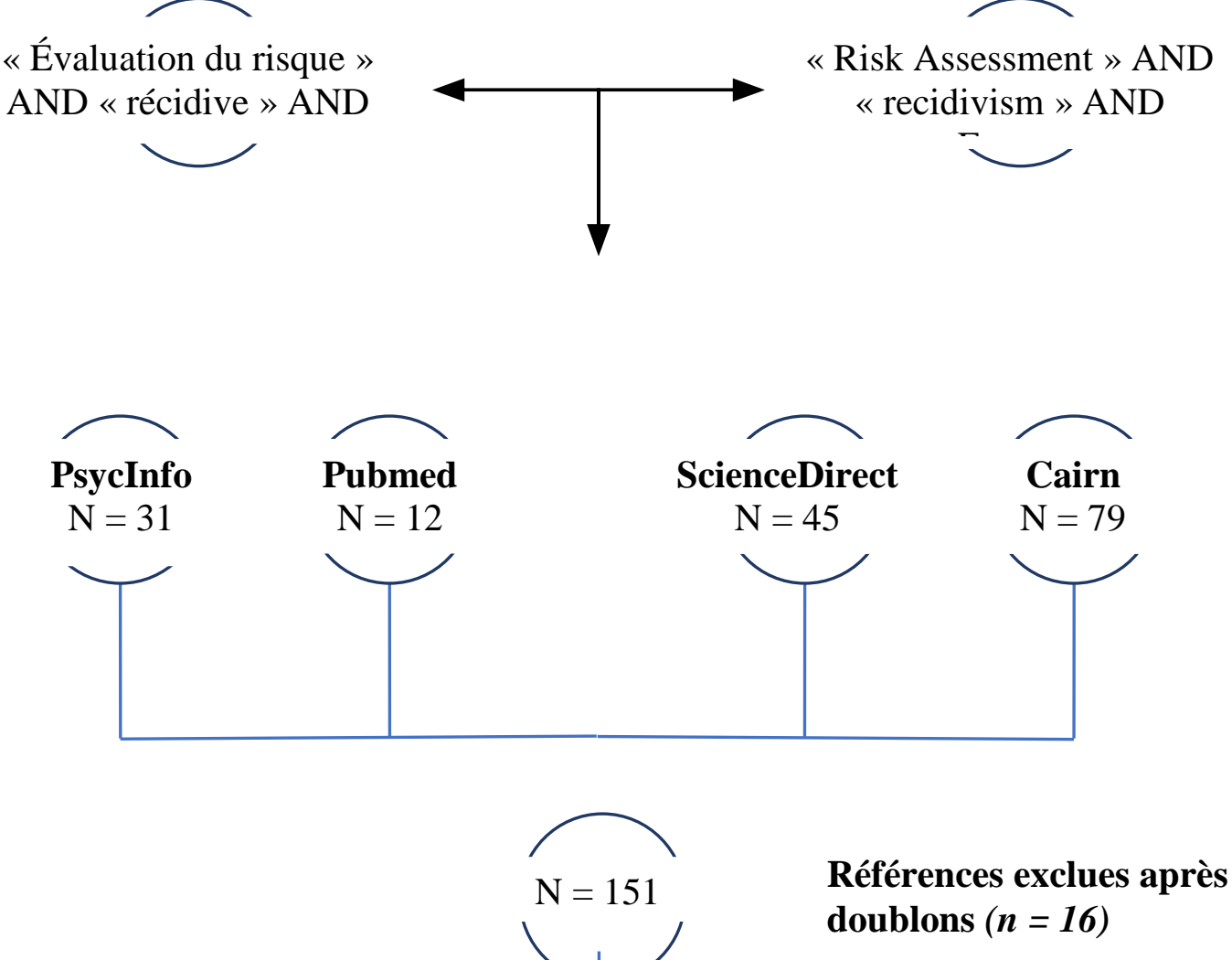

Références exclues après lecture des résumés $n=126$

- Population inadaptée $(n=37)$ :

- Population non délictuelle $(n=13)$

- Population étrangère $(n=24)$

$$
\mathrm{N}=25
$$

- Thématique hors sujet $(n=82)$

- Prise en charge $(=28)$

- Risque suicidaire $(n=8)$

- Risque somatique ( $n=9)$

- Dangerosité

psychiatrique/explications 
Références exclues après lecture des articles complets $(n=2)$ :

- Prise en charge $(n=1)$

- Population étrangère $(n=1)$

$\mathbf{N}=\mathbf{2 3}$ articles

REFERENCES FINALES 
Tableau 1. Principaux résultats des études sélectionnées

\begin{tabular}{|c|c|c|c|c|c|c|}
\hline \multirow[b]{2}{*}{ Références } & \multirow[b]{2}{*}{$\begin{array}{l}\text { Types de } \\
\text { document }\end{array}$} & \multicolumn{4}{|c|}{ Thématiques abordées dans les articles } & Principaux résultats \\
\hline & & $\begin{array}{l}\text { Les } \\
\text { craintes } \\
(\mathrm{N}=10)\end{array}$ & $\begin{array}{l}\text { Les } \\
\text { avantage } \\
\text { s }(\mathrm{N}=5)\end{array}$ & $\begin{array}{l}\text { Vers un } \\
\text { système } \\
\text { hybride } \\
? \\
(\mathrm{~N}=6)\end{array}$ & $\begin{array}{l}\text { Des } \\
\text { travaux } \\
\text { du } \\
\text { risque } \\
\text { en } \\
\text { populati } \\
\text { on } \\
\text { français } \\
\text { e }(\mathrm{N}=3)\end{array}$ & \\
\hline Baratta (2011) & $\begin{array}{l}\text { Article de } \\
\text { réflexion }\end{array}$ & $x$ & $x$ & $\times$ & & $\begin{array}{l}\text { - Prévalence de la méthode du JPNS } \\
\text { - Mise en œuvre d'une évaluation semi-structurée en France }\end{array}$ \\
\hline Baratta (2013) & $\begin{array}{l}\text { Lettre à la } \\
\text { rédaction }\end{array}$ & & $x$ & & & $\begin{array}{l}\text { - Pertinence des échelles de risque structurée } \\
\text { - Avantages à l'utilisation des échelles structurées : scientificité et objectivité }\end{array}$ \\
\hline $\begin{array}{l}\text { Benbouriche et al. } \\
\text { (2015) }\end{array}$ & $\begin{array}{l}\text { Article de } \\
\text { réflexion }\end{array}$ & & $x$ & $\times$ & & $\begin{array}{l}\text { - Définition de la récidive } \\
\text { - Rappel et pertinence du modèle RBR }\end{array}$ \\
\hline Bertsch et al. (2017) & $\begin{array}{l}\text { Recherche } \\
\text { quantitative }\end{array}$ & & & & $\times$ & $\begin{array}{l}\text { - Mise en place d'une étude sur l'évaluation structurée du risque de récidive en } \\
\text { population française }\end{array}$ \\
\hline
\end{tabular}




\begin{tabular}{|c|c|c|c|c|c|c|}
\hline & & & & & & - Échelles de risque utilisées : RSVP, HCR-20 et LSCMI \\
\hline Bessoles (2004) & $\begin{array}{l}\text { Article de } \\
\text { réflexion }\end{array}$ & $x$ & & & $x$ & $\begin{array}{l}\text { - Définition de la récidive } \\
\text { - Rappel des taux de récidive et des différents traitements } \\
\text { - Critiques des outils actuariels }\end{array}$ \\
\hline Blatier et al. (2012) & $\begin{array}{l}\text { Recherche } \\
\text { quantitative }\end{array}$ & & & & $\times$ & - Facteurs liés à la récidive : empathie, estime de soi, attitudes sociocognitives \\
\hline Coutanceau (2012) & $\begin{array}{l}\text { Article de } \\
\text { réflexion }\end{array}$ & & & $x$ & & $\begin{array}{l}\text { - Définitions de dangerosité criminologique et psychiatrique } \\
\text { - Approches dimensionnelle et catégorielle de la dangerosité criminologique } \\
\text { - Analyse qualitative et quantitative de l'évaluation du risque à mettre en place en } \\
\text { France }\end{array}$ \\
\hline Dailliet (2008) & $\begin{array}{l}\text { Article de } \\
\text { réflexion }\end{array}$ & & $x$ & & & $\begin{array}{l}\text { - Historique de la conception de la Justice en matière d'expertise } \\
\text { - Avantages à l'utilisation des échelles } \\
\text { - Biais des méthodes actuarielles }\end{array}$ \\
\hline Ducro et al. (2012) & $\begin{array}{l}\text { Recherche } \\
\text { quantitative }\end{array}$ & & & & $\times$ & $\begin{array}{l}\text { - Évaluation du risque par Static-99 et SORAG d'AICS } \\
\text { - Comparaisons AICS d'adultes vs d'enfants, intra vs extrafamiliaux }\end{array}$ \\
\hline Goumilloux (2016) & $\begin{array}{l}\text { Recherche } \\
\text { quantitative }\end{array}$ & $\times$ & & & & $\begin{array}{l}\text { - Contexte judicaire actuel de l'évaluation structurée du risque } \\
\text { - Principaux facteurs de risque et de protection } \\
\text { - Nécessaire de prise en compte de la culture française dans l'arrivée de l'évaluation } \\
\text { structurée du risque }\end{array}$ \\
\hline Gravier et al. (2012) & $\begin{array}{l}\text { Article de } \\
\text { réflexion }\end{array}$ & $\times$ & & $x$ & & $\begin{array}{l}\text { - Craintes de l'arrivée des échelles en France } \\
\text { - Intérêt d'une évaluation clinique du risque et recommandations de bonnes pratiques }\end{array}$ \\
\hline $\begin{array}{l}\text { Guay et Lafortune } \\
\text { (2015) }\end{array}$ & $\begin{array}{l}\text { Article de } \\
\text { réflexion }\end{array}$ & & & $x$ & & - Implantation de l'évaluation en France \\
\hline Guay et al (2015) & $\begin{array}{l}\text { Article de } \\
\text { réflexion }\end{array}$ & $x$ & $\times$ & $x$ & & $\begin{array}{l}\text { - Définition des étapes de l'évaluation du risque } \\
\text { - Imperfection des mesures de risque }\end{array}$ \\
\hline
\end{tabular}




\begin{tabular}{|c|c|c|c|c|c|c|}
\hline & & & & & & $\begin{array}{l}\text { - Nécessité d'évaluer les erreurs commises lors du JPNS } \\
\text { - Différentes générations d'échelles de risque } \\
\text { - Exemples d'échelles }\end{array}$ \\
\hline $\begin{array}{l}\text { Harcourt } \\
\text { (2011a) }\end{array}$ & $\begin{array}{l}\text { Article de } \\
\text { réflexion }\end{array}$ & $x$ & & & & - Généalogie du tournant actuariel et les risques pour la France \\
\hline Harcourt (2011b) & $\begin{array}{l}\text { Article de } \\
\text { réflexion }\end{array}$ & $x$ & & & & $\begin{array}{l}\text { - Critique du caractère scientifique de l'évaluation du risque } \\
\text { - Coût de l'évaluation du risque sur la société } \\
\text { - Impact négatif de l'évaluation du risque en matière de réinsertion }\end{array}$ \\
\hline $\begin{array}{l}\text { Mouchet-Mages, et al. } \\
\text { (2017) }\end{array}$ & $\begin{array}{l}\text { Retour sur } \\
\text { pratique }\end{array}$ & & $\times$ & $\times$ & & $\begin{array}{l}\text { - Utilisation des échelles dans cadre d'une pratique clinique } \\
\text { - Retours sur } 10 \text { dossiers de patients évalués (HCR-20 et RSVP) }\end{array}$ \\
\hline Moulin et al. (2012) & $\begin{array}{l}\text { Article de } \\
\text { réflexion }\end{array}$ & $\times$ & $\times$ & & & $\begin{array}{l}\text { - Évolution législative de la prévention de la récidive en France } \\
\text { - Historique des concepts de dangerosité et de risque } \\
\text { - Craintes liées à l'arrivée de l'évaluation structurée du risque en France : } \\
\text { - Instauration d'objectivité dans l'évaluation du risque }\end{array}$ \\
\hline Pham et al. (2005) & $\begin{array}{l}\text { Recherche } \\
\text { quantitative }\end{array}$ & & & & $x$ & $\begin{array}{l}\text { - Évaluation de la validité convergente entre la PCL-R, la HCR-20 } \\
\text { - Liens entre les syndromes cliniques, les troubles de la personnalité du DSM-IV et la } \\
\text { HCR-20 et la PCL-R }\end{array}$ \\
\hline Raoult (2014) & $\begin{array}{l}\text { Article de } \\
\text { réflexion }\end{array}$ & $x$ & & & & - Critiques de la recherche sur la récidive \\
\hline Rio et al. (2017) & $\begin{array}{l}\text { Recherche } \\
\text { quantitative }\end{array}$ & & & & $\times$ & $\begin{array}{l}\text { - Évaluation du risque de } 55 \text { détenus (Static-99, VRAG, SORAG et PCL-R + } \\
\text { entretiens avec des experts sur le niveau de risque des évalués) }\end{array}$ \\
\hline Senon et al. (2009) & $\begin{array}{l}\text { Article de } \\
\text { réflexion }\end{array}$ & & & $x$ & & $\begin{array}{l}\text { - Historique du changement de la politique pénale en Amérique du Nord et en Europe } \\
\text { - Description des échelles HCR-20 et VRAG } \\
\text { - Intérêt du développement des outils en France }\end{array}$ \\
\hline
\end{tabular}




\begin{tabular}{|c|c|c|c|c|c|}
\hline $\begin{array}{l}\text { Vanderstukken et al. } \\
\text { (2011) }\end{array}$ & $\begin{array}{l}\text { Article de } \\
\text { réflexion }\end{array}$ & $x$ & $x$ & $x$ & $\begin{array}{l}\text { - Climat d'insécurité autour de la notion de dangerosité } \\
\text { - Rappel des risques de la poursuite du risque } 0 \text { sur le système pénal et sanitaire et } \\
\text { des chiffres de la récidive } \\
\text { - Mesures de rétention de sureté } \\
\text { - Implantation des échelles de risque comme avantages à la prise en charge }\end{array}$ \\
\hline Voyer \& Senon 2012) & $\begin{array}{l}\text { Article de } \\
\text { réflexion }\end{array}$ & $x$ & $x$ & $x$ & $\begin{array}{l}\text { - Historique du développement de l'évaluation structurée du risque } \\
\text { - Critiques des méthodes actuarielles } \\
\text { - Outils de jugement clinique structuré }\end{array}$ \\
\hline
\end{tabular}

